\title{
The Peripheral Distribution and Central Projections of the Sensory Rami of the Facial Nerve in Goldfish, Carassius auratus
}

\author{
R.L. PUZDROWSKI
}

Division of Biological Sciences, The University of Michigan, Ann Arbor, Michigan 48109

\begin{abstract}
Taste buds in goldfish and other cyprinids are found not only within the oropharyngeal cavity but also scattered over the external body surface. The external taste buds are innervated by branches of the facial nerve that terminate centrally in an enlargement of the medulla termed the facial lobe. The peripheral distribution and areas of innervation of the rami of the facial sensory nerve were determined by using a modification of the Sihler technique and by examination of a Bodian-stained head series. The central projections of individual rami of the facial sensory nerve were traced by means of the horseradish peroxidase (HRP) technique.

Fibers of the facial sensory nerve distribute over the head and trunk via nine rami. The supraorbital ramus distributes fibers to taste buds above the eye. The palatine, maxillary, and mandibular rami innervate taste buds of the rostral palate, upper lip, and lower lip, respectively. The three rami of the hyomandibular trunk innervate taste buds on the operculum, branchiostegal rays, and in the lower cheek region. A facial recurrent ramus was also found that distributes fibers to taste buds on the trunk and pectoral fin via two rami, the lateral recurrent ramus and pectoral recurrent ramus.

The facial sensory rami map somatotopically on the facial lobe. Overall, the projections follow an anteroposterior orientation with the long axis of the body tilted slightly ventrally. The lips and rostral palate make up a disproportionately large portion of the map, taking up nearly the entire ventral extent of the lobe. The trunk and pectoral fin regions map broadly across the dorsal portion of the lobe. Further, projections to the nucleus of the descending trigeminal tract were observed with labeling of the supraorbital, maxillary, and mandibular rami, and the rami of the hyomandibular trunk. Projections to the facial motor nucleus were also observed with labeling of maxillary and mandibular rami, perhaps indicating a monosynaptic reflex arc. These projections have not been reported in previous studies on the teleostean facial taste system.
\end{abstract}

Key words: facial lobe, cyprinid, taste bud, somatotopy, horseradish peroxidase

Both cyprinid and silurid teleosts have a large number of taste buds distributed not only within the mouth and pharynx but also across the entire body surface (Herrick, '03; Atema, '71). The taste buds of the pharynx and caudal palate are innervated by the IXth and Xth cranial nerves, whereas those of the anterior palate and body surface are innervated by the VIIth cranial nerve (Herrick, '01).

The primary brainstem targets of these nerves, in accordance with the number of taste buds, constitute large lobes and have been described in both cyprinids and silurids (Finger, '76; Morita et al., '80). The VIIth cranial nerve projects mainly to the facial lobe, whereas the IXth and Xth cranial nerves project to the glossopharyngeal and vagal lobes, respectively.
In silurids, the facial lobes are divided into three lobules arranged mediolaterally (Herrick, '05; Finger, '76). The peripheral taste buds are represented topographically on these lobules; the trunk taste buds are represented on the lateral lobule, the nasal barbel buds on the intermediate lobule, and the maxillomandibular buds are represented on the medial lobule (Finger, '76). This topographic organization has been confirmed electrophysiologically (Marui and Caprio, ' 82 ).

While there is electrophysiological evidence of somatotopic maps on the facial lobe in goldfish (Peterson, '72) and

Accepted September 11, 1986. 
carp (Marui, '77), these two studies do not agree on the orientation of the somatotopy. Marui (77), working on the carp, Cyprinus carpio, found the anteroposterior body axis to be represented dorsoventrally on the facial lobe. Peterson ('72), on the other hand, found the anteroposterior body axis in goldfish (Carassius auratus) to be represented along the anteroposterior axis of the lobe. Recent anatomical studies have yielded further contradictory results. Kiyohara et al. ('85), using horseradish peroxidase (HRP) application to selected branches of the facial nerve in $C$. carpio, demonstrated that the branches project topographically, with the anteroposterior body axis represented along the anteroposterior axis of the lobe. Morita et al. ('80), utilizing degeneration techniques, found that a somatotopic organization is not clearly distinguishable in the facial lobe of the crucian carp, Carassius carassius, a species closely related to goldfish. Thus, a clear and complete somatotopic representation of the peripheral facial taste system on the facial lobe in cyprinids remains to be demonstrated.

This study was undertaken to elucidate the topographic projections of the facial sensory rami in the goldfish, Carassius auratus, by utilizing horseradish peroxidase (HRP) techniques. An abstract of this work has been published previously (Puzdrowski, '84).

\section{MATERIALS AND METHODS}

Forty-seven adult goldfish, Carassius auratus, were used in this study. The animals ranged in size from 8 to $12 \mathrm{~cm}$ in total length. In all surgical or perfusion procedures the animals were anesthetized with tricane methane sulfonate (0.025\% solution, Sigma, St. Louis, MO).

The peripheral distribution of the facial sensory rami were determined in two specimens by using a modification of the Sihler technique (Freihofer, '66). Specimens processed by this technique are rendered transparent except for the nerves, which are stained a dark purple. The intracranial course of these rami was studied in one specimen perfused with $0.1 \mathrm{M}$ phosphate buffer (pH 7.4) and $10 \%$ formalin. A few drops of $1 \%$ osmium tetroxide were applied to the cranial nerves to enhance visibility.

The distribution of the taste buds innervated by the facial sensory rami and the general area of innervation of each ramus were determined by examination of a normal, Bodian-stained head series sectioned transversely at $10 \mu \mathrm{m}$. Since in their peripheral course the facial rami are closely associated with the rami of the trigeminal and lateral line nerves, the peripheral distribution of these latter cranial nerves was also examined.

All surgical procedures were carried out with the aid of a stereozoom operating microscope. Following anesthetization, the fish was wrapped in moistened cheesecloth and individual sensory rami of the VIIth cranial nerve were exposed and transected. A Gelfoam pledget soaked in $40 \%$ HRP (Sigma VI) was placed on the proximal stump of the transected branch, and the wound was closed with Histoacryl (Tri Hawk International, Distributors, Montreal, Quebec). The location of HRP application to each peripheral ramus is illustrated in Figure 1A. In order to label the rami that course along the walls of the orbit (ROS, PAL, MAN, MAX, see Fig. 1A) the eyeball was retracted, the nerve transected and filled with HRP, and the eyeball then replaced and covered with histoacryl. The hyomandibular trunk was transected and filled with HRP at a point in the rostrodorsal wall of the opercular cavity where it courses superficially before entering the hyomandibular bone. The
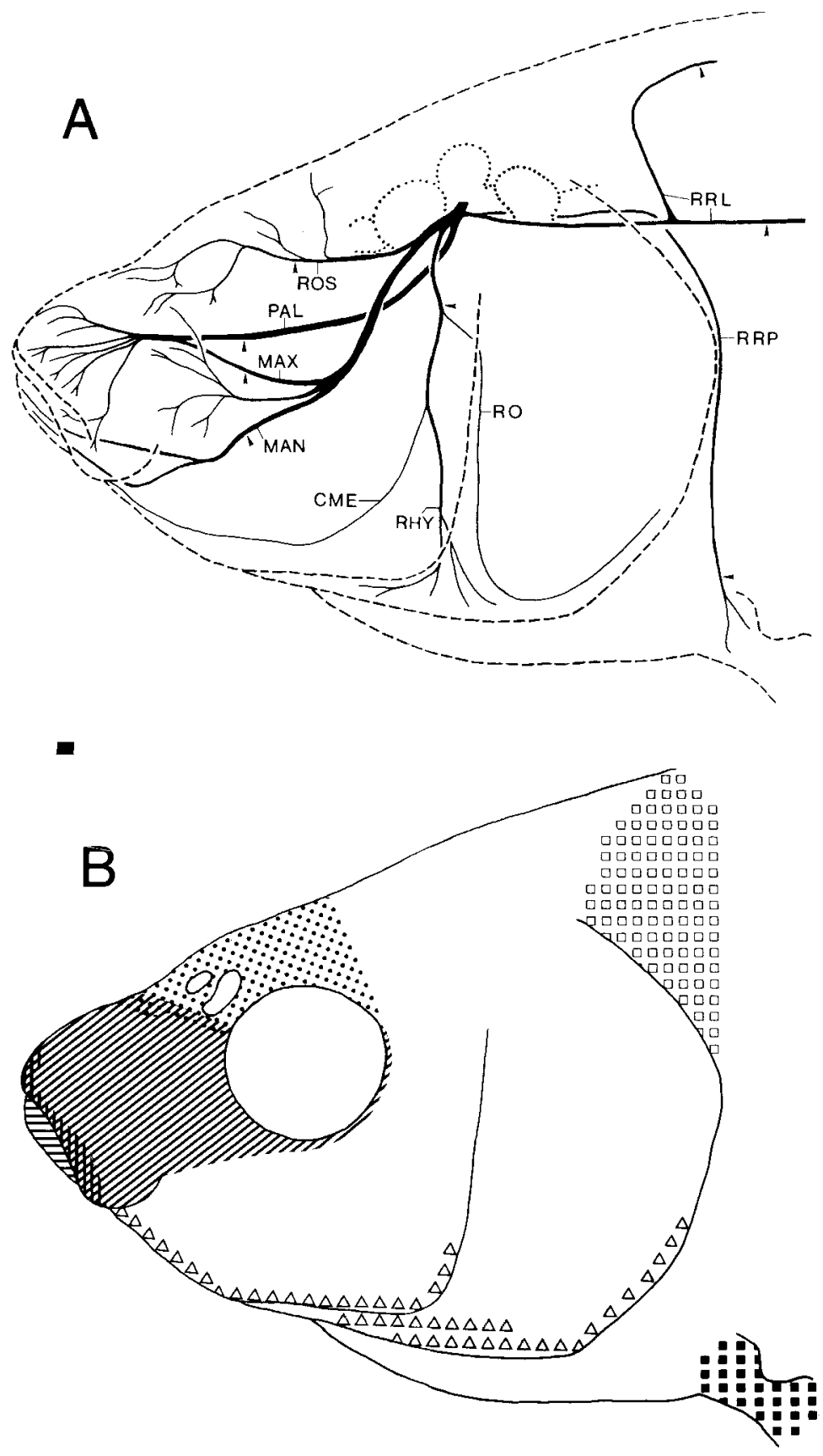

Fig. 1. Lateral view of the head and anterior trunk of Carassius auratus. A: Peripheral distribution of the rami of the facial taste system in the head region of the goldfish. B: The areas of taste bud innervation by the rami of the facial sensory nerve. The region innervated by ROS is represented by stippling; MAX by oblique hatching; PAL by vertical hatching; MAN by horizontal hatching; HYO by open triangles; RRL by open squares; RRP by filled squares. Taste bud density is not indicated in this figure. Bar scale equals $1 \mathrm{~mm}$.

remaining two rami, the lateral recurrent ramus and the pectoral recurrent ramus, are quite superficial and were easily exposed by making a small slit in the skin and transecting and filling the nerve which lay beneath.

Following a survival time of $7-14$ days at $22-26^{\circ} \mathrm{C}$ the animals were reanesthetized and transcardially perfused 
with cold $0.1 \mathrm{M}$ phosphate buffer, followed by $4 \%$ glutaraldehyde in phosphate buffer. Following a 1-hour postfixation in $4 \%$ glutaraldehyde in $20 \%$ sucrose phosphate buffer, the brains were washed for 8 hours in $20 \%$ sucrose-buffer, the meninges were removed, and the brains were embedded in gelatin. The gelatin blocks were fixed for an additional 4 hours in $4 \%$ glutaraldehyde in $20 \%$ sucrose-phosphate buffer. The blocks were then rinsed in $20 \%$ sucrose-phosphate buffer for 8 hours to remove any excess fixative. Forty micron transverse sections of the gelatin-embedded brains were cut on a sliding microtome, collected in $0.1 \mathrm{M}$ phosphate buffer, and stored at $4^{\circ} \mathrm{C}$. The sections were processed according to the Mesulam ('78) tetramethylbenzidine (TMB) protocol, or by the Hanker-Yates protocol (Hanker et al., '77) for visualization of HRP. Sections processed with TMB were examined with both bright field and polarized light.

A total of 44 experimental cases were used in this study. Bilateral labelings, in which different rami of the facial nerve were labeled on each side of the animal, were done in order to compare the relative positions of terminal fields of individual rami. Of the 44 experimental cases, there were seven cases each of the superficial ophthalmic ramus and the lateral recurrent ramus; four cases of the pectoral recurrent ramus; five cases of the infraorbital trunk (the mandibular ramus and the maxillary ramus, combined), in addition to four cases in which the maxillary ramus was labeled on one side and the mandibular ramus on the other
Abbreviations

\section{$\mathrm{Cb} \quad$ Cerebellum}

CC Cerebellar crest

CME Cutaneous mandibular branch of external mandibular ramus

DTV Descending trigeminal tract

FL Facial lobe

GL Glossopharyngeal lobe

IR Inferior reticular formation

IV Fourth ventricle

MA Mauthner axon

MAN Mandibular ramus

MAX Maxillary ramus

MLF Medial longitudinal fasiculus

MR Medial reticular formation

PAL Palatine ramus

RHY Hyoid ramus

RO Opercular ramus

ROS Superficial ophthalmic ramus

RRL Lateral recurrent ramus

RRP Pectoral recurrent ramus

RSVII Root of the facial nerve

SG Secondary gustatory tract

VAVL Valvula of the cerebellum

VL Vagal lobe

XN Vagal nerve

side; five cases in which the infraorbital trunk was filled on one side and the superficial ophthalmic ramus was filled on the opposite side; six cases of the palatine ramus, one in which the infraorbital trunk was filled on the opposite side,
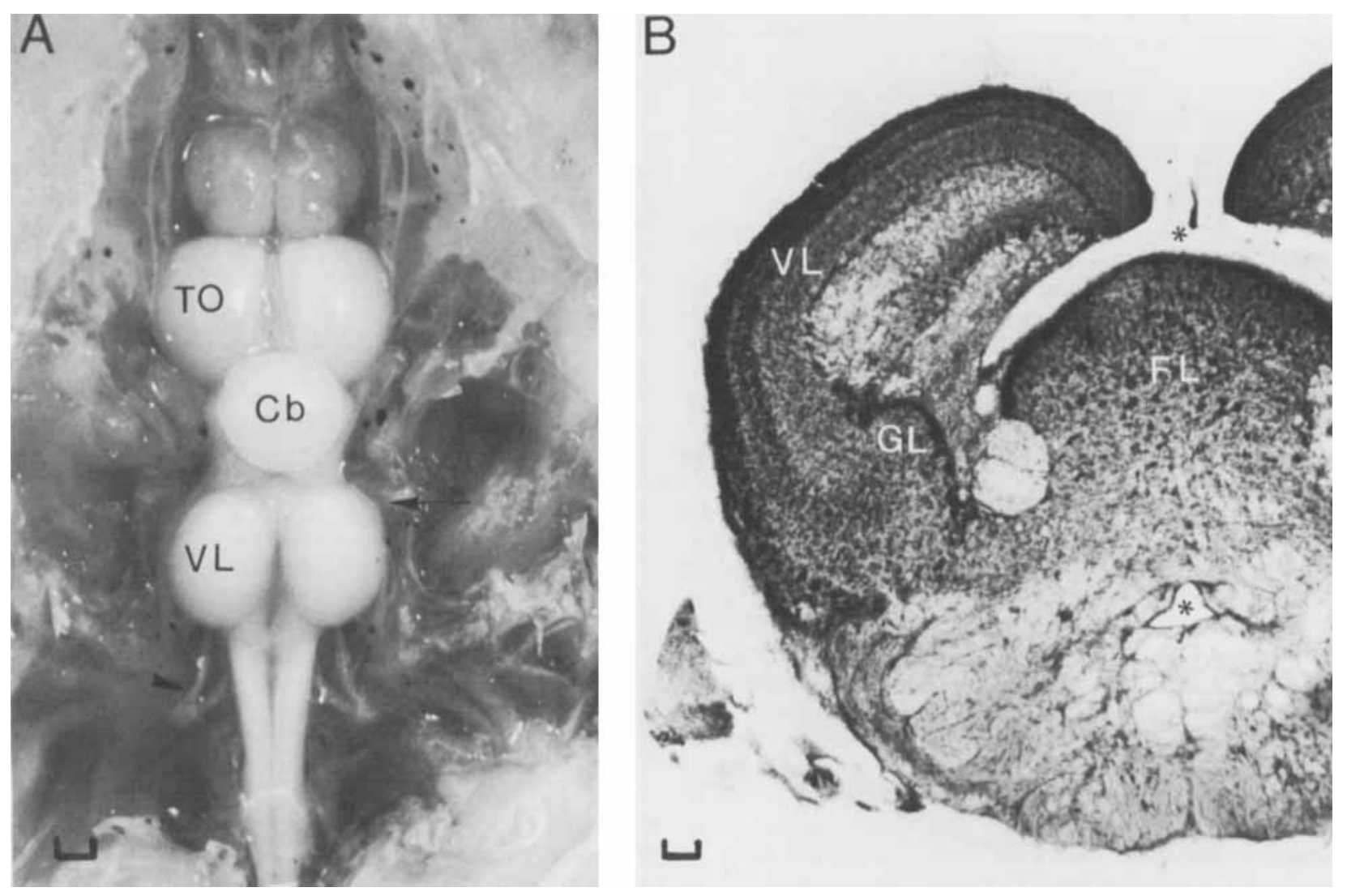

Fig. 2. A: Dorsal view of goldfish brain. Arrow marks approximate level of panel B. Arrowhead indicates recurrent ramus joining with the first spinal root. B: Photomicrograph of a transverse section through the level of the facial, glossopharyngeal and vagal lobes. Note the lamination of the vagal lobes and the cell clusters scattered throughout the facial lobe. Asterisks mark fourth ventricle. Bar scale for A equals $0.5 \mathrm{~cm}$; B equals 0.02 $\mathrm{mm}$. 
and one case in which the superficial ophthalmic ramus was filled on the opposite side; and five cases of the hyomandibular trunk, three in which the infraorbital trunk was filled on the opposite side.

\section{RESULTS}

The most prominent feature of the brainstem in cyprinids is the great enlargement of the gustatory centers, the facial, glossopharyngeal, and vagal lobes (FL, GL, VL, Fig. 2). The facial lobe is believed to represent the hypertrophy of the rostral part of the visceral sensory column (Herrick, '03). The single-lobe structure results from the fusion at the midline of the enlarged cephalic ends of the right and left visceral sensory columns.

The cytoarchitecture of the gustatory centers in cyprinids has been previously described in detail (Herrick, '05; Morita et al., '83). The organization of the facial lobe is simple compared to that of the vagal lobes (Fig. 2B). The facial lobe is formed dorsally by small granular cells, which aggregate to form an outer layer facing the fourth ventricle. The center of the lobe is composed of scattered cells of several different sizes (Morita et al., '83). In some instances the cells of the center of the lobe are grouped into small clusters, as described by Herrick ('05). These clusters do not appear to be organized in any particular pattern in the facial lobe of goldfish.

\section{The distribution of external taste buds and facial sensory rami}

External taste buds were recognized on the basis of the description by Reutter ('78) of the barbel taste buds of the bullhead catfish (Ictalurus). The external taste buds, like taste buds of the pharynx, are pear-shaped structures, situated on a dermal papilla which is pierced by the innervating sensory fibers. The cells of the sensory epithelium of a taste bud extend across the epithelium from the basal lamina to the skin surface. The nuclei of the sensory cells of a taste bud are fusiform with the long axis of the nucleus oriented perpendicular to the skin surface.

The distribution of the external taste buds and associated nerves in goldfish are illustrated in Figure 1. External taste buds were found to be most abundant in the region of the lips and rostral to the eye. The pectoral fin was not examined for taste buds; however, the region does receive fibers from the facial nerve (RRP, Fig. 1A).

As in other ostariophysians (Herrick, '01; Luiten, '75) the trigeminal, facial, and anterior lateral line ganglia in goldfish fuse early in embryogenesis to form a loose ganglionic plexus. It was possible to distinguish the components of the ganglionic complex by tracing the cranial nerve roots of the trigeminal, facial, and anterior lateral line nerves to their respective portion of the complex. The extensive facial ganglion constitutes the entire caudal and ventrolateral portion of the ganglionic plexus, with the ventralmost portion of the facial ganglion lying beneath the brain.

The facial ganglion distributes fibers over the head and trunk region through nine rami as illustrated in Figure 1A. The nomenclature used is that of Herrick ('01).

The superficial ophthalmic ramus. The facial fibers of the superficial ophthalmic ramus (ROS, Fig. 1A) arise from the dorsorostral part of the ganglionic complex in common with a bundle of trigeminal fibers. This nerve complex courses rostrally along the optic tectum and exits from the braincase along with the trochlear nerve through a foramen lateral to the rostral pole of the tectum. After emerging from the cranium, this ramus courses rostrally along with the trochlear nerve and a branch of the anterior lateral line nerve. In its course along the roof of the orbit, the superficial ophthalmic ramus distributes fibers to the taste buds located in the skin dorsal to the eye. Upon reaching the front of the orbit, the superficial ophthalmic ramus passes through a foramen in the frontal bone to distribute fibers to taste buds in the region of the nasal aperture (Fig. 1B).

The rami of the infraorbital trunk. The infraorbital trunk is composed of fibers from the dorsal ganglion of the anterior lateral line nerve, the trigeminal ganglion, and the facial ganglion. Peripherally the infraorbital trunk divides into two rami, the maxillary ramus and the mandibular ramus. Both carry facial sensory fibers. The facial fibers of the maxillary ramus and mandibular ramus arise from the rostroventral part of the ganglion and are immediately joined by fibers from the dorsal ganglion of the anterior lateral line nerve. This nerve trunk, commonly referred to as the infraorbital trunk, leaves the braincase through a large foramen lateral to the optic tectum and courses ventrally and then rostrally along the caudal wall of the orbit. As the nerve trunk passes down along the caudal wall of the orbit, facial fibers are given off to the external taste buds located along the caudal edge of the orbit (Fig. 1B). In addition, there is a small branch from the infraorbital trunk that courses across the cheek region ventrally and anastomoses with the hyomandibular trunk (not shown in Fig. 1B). The exact nature of this branch is uncertain. At approximately the middle of the orbit, the facial fibers of the infraorbital trunk divide into the mandibular ramus (MAN, Fig. 1A) and the maxillary ramus (MAX, Fig. 1A). The fibers of the mandibular ramus course ventrally, medial to the larger mass of the adductor mandibulae distributing fibers to the floor of the mouth. The bulk of the facial fibers of the mandibular ramus distribute to taste buds located in the region of the lower lip. After separating from the mandibular ramus, the fibers of the maxillary ramus divide again into a medial and lateral branch. The lateral branch distributes fibers to taste buds in the region rostral to the eye and ventral to the nares. The medial branch courses rostromedially to anastomose with the palatine ramus. This nerve complex then divides into five bundles that course rostrally distributing fibers to the rostral palate and upper lip.

The rami of the hyomandibular trunk. The facial fibers of the hyomandibular trunk originate from the facial ganglion caudal and ventral to those of the infraorbital trunk. Accompanied by lateral line fibers from the ventral ganglion of the anterior lateral line nerve, the hyomandibular fibers exit from the braincase immediately and course rostrolaterally a short distance before being joined by a bundle of trigeminal fibers. This nerve trunk, the hyomandibular trunk, continues laterally and then turns ventrally to course superficially along the rostrodorsal wall of the opercular cavity before passing through the hyomandibular bone to course ventrally.

Midway in its course through the hyomandibular bone, the hyomandibular trunk gives off the fibers of the opercular ramus (RO, Fig. 1A). This ramus exits from the hyomandibular bone to course ventrally along the subopercle, supplying the taste receptors along the ventral edge of the operculum. 
The rest of the hyomandibular trunk continues to course ventrally through the hyomandibular bone. The trunk exits from the hyomandibular bone through a foramen at its ventral edge and divides into two rami, the hyoid ramus (RHY, Fig. 1A) and the external mandibular ramus. The hyoid ramus courses ventrally, medial to the preopercle to supply taste buds in the skin on the interopercle and branchiostegal rays (Fig. 1B). The external mandibular ramus courses rostrally along the dorsal edge of the preopercle a short distance before dividing into two branches of approximately equal diameter. One of the branches passes ventrally and enters the alveolar canal of the preopercle supplying neuromasts of the operculomandibular canal. The other branch, termed the cutaneous mandibular branch of the external mandibular ramus (CME, Fig. 1A) is composed of both lateral line and facial sensory fibers. The cutaneous mandibular ramus remains superficial, coursing rostrally along the dorsal edge of the preopercle supplying taste buds and free neuromasts in the vicinity of the operculomandibular canal. No taste buds inside the mouth or on the lower lip were found to be innervated by fibers originating from the rami of the hyomandibular trunk.

The palatine ramus. The palatine ramus (PAL, Fig. 1A) leaves the ventralmost part of the ganglion, immediately caudal to the facial fibers of the hyomandibular trunk. The palatine ramus exits from the braincase through a foramen ventrolateral to the inferior lobe of the hypothalamus. The fibers continue ventrally to the parasphenoid bone and then turn rostrally to course along the medial wall of the orbit accompanied by a branch of the oculomotor nerve. After passing through a foramen in the rostral part of the orbit, the palatine ramus begins to distribute fibers to taste recep tors of the palatal mucosa. The bulk of the fibers of the palatine ramus continue rostrally, where they are joined by a branch of the maxillary ramus of the infraorbital trunk. The remaining course of this nerve complex has already been described.

The facial recurrent ramus. The course of the recurrent ramus follows the general pattern for cyprinids as described by Freihofer ('63). The recurrent ramus originates from the posteriormost part of the facial ganglion as a single branch and divides immediately into the pectoral recurrent ramus and the lateral recurrent ramus (RRP and RRL, respectively. Fig. 1A). The pectoral recurrent ramus courses caudally, medial to the roots of the VIIIth, IXth, and Xth cranial nerves, and joins with the first spinal nerve. This nerve complex courses ventrally along the medial side of the pectoral girdle to the pectoral fin. The lateral recurrent ramus courses caudally, lateral to the roots of the VIIIth cranial nerve. Before exiting from the braincase it anastomoses with the posterior lateral line nerve. This nerve complex passes out of the cranium through the posterior vagal foramen and courses superficially along the body. The fibers of this ramus are distributed to taste receptors of the trunk.

\section{The central projections of the facial sensory rami}

Because fibers from cranial nerve $V$, the anterior lateral line nerve, and the posterior lateral line nerve intermingle with the facial rami to form complex nerves, peripheral HRP fills of the facial rami result in labeling not only facial fibers, but all fibers associated in the complex. Intracranially, the fibers of each nerve segregate and enter the brainstem through distinctly separate roots. It was, therefore, possible to trace all fibers to their roots of origin and thus identify the source of any particular projection. Afferent fibers in the lateral line and trigeminal roots were observed with the labeling of all facial rami except the palatine ramus. Most of the trigeminal fibers enter the descending trigeminal tract and descend through the medulla to end in the medial funicular nucleus (Puzdrowski, '85). Following labeling of the infraorbital trunk a few fibers were observed coursing from the descending trigeminal tract to the ventral aspect of the facial lobe. In no case were fibers originating from the lateral line roots observed to project to the facial lobe. Additionally, the efferent cells of the anterior lateral line nerve and the posterior lateral line nerve and the trigeminal and facial motor cells were labeled in this study. The organization of these centers will be described in a subsequent paper.

The facial sensory fibers enter the medulla as a single, large root that courses mediocaudally to the midline. Upon reaching the midline the fibers turn caudally and descend, entering the facial lobe as a large dorsolateral bundle accompanied by two smaller ventral bundles. These bundles course along the lateral aspect of the lobe. The dorsolateral bundle distributes fibers to the dorsal and ventromedial portion of the lobe. The ventral bundles distribute fibers to the ventral portion of the lobe.

The individual rami of the sensory component of the facial nerve project in a topographic manner onto the facial lobe (Fig. 3). The projections of the rami are for the most part ipsilateral.

Following application of HRP to the superficial ophthalmic ramus, labeled fibers were observed to enter the facial lobe mainly through the large dorsolateral bundle. Light labeling was also observed in the fibers of the ventral bundles. Except for the rostralmost portion (Fig. $3 \mathrm{~A}$ ), all projections of the superficial ophthalmic ramus were ipsilateral. The terminal field of this ramus lies in the rostral two-thirds of the facial lobe (Fig. 3A-D), extending from the rostralmost pole of the lobe to the level of the entrance of the rostral root of the vagal nerve. The caudal part of the terminal field of the superficial ophthalmic ramus lies in the dorsal-intermediate portion of the lobe ventral to the projection field of the lateral recurrent ramus and dorsal to that of the maxillary ramus (Fig. 3B-D). The majority of the fibers entering the lobe through the ventral bundles project, along with those of the dorsal bundle, to the termination area described above. However, at the level of the rostral pole of the vagal lobes, a few fibers of the ventral bundle project ventrally through the ventrolateral portion of the lobe and end in the nucleus of the descending trigeminal tract.

The projections of the palatine ramus and the rami of the infraorbital trunk rami (the maxillary and mandibular rami) occupy most of the entire ventral portion of the facial lobe (Figs. 3A-E, 4). The afferent fibers of the maxillary ramus reach the lobe mainly through the dorsolateral bundle of the facial sensory root and terminate in the ventrodorsal portion of the lobe (Fig. 3B-E). The most rostral projection of this ramus crosses the midline (Fig. 3B). The terminal field of the maxillary ramus overlaps dorsally with the terminal field of the superficial ophthalmic ramus and ventrally with the terminal field of the palatine ramus. The afferent fibers of the mandibular ramus enter the ventralmost portion of the lobe mainly through the ventral bundles of the facial sensory root and terminate in the ventralmost portion of the facial lobe (Fig. 4B). At the level of the rostral pole of the vagal lobes, the fibers penetrate 
Fig. 3. Schematic representation of the relative positions of the terminal fields of the facial sensory rami on the facial lobe. Section A is most rostral, F most caudal. Key to symbols is at the lower left of the figure. Midsagittal section in the upper right indicates the level of each transverse section.
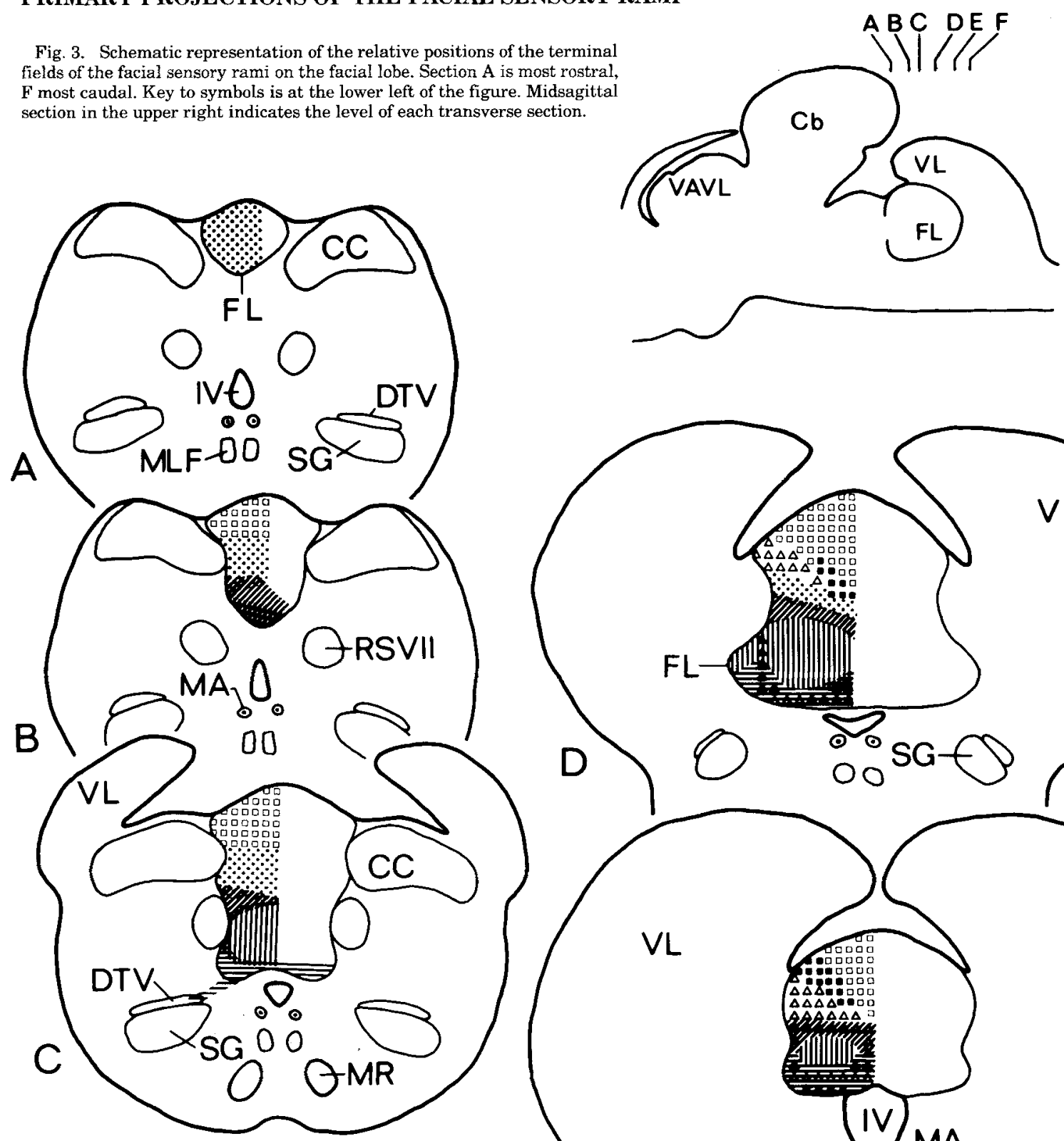

$\longrightarrow 1 \mathrm{Vt}$

A

A $\mathrm{ML}$

R ROS $\left[\begin{array}{ll}\Delta \Delta \Delta \Delta \Delta \Delta \\ \Delta \Delta \Delta \Delta \Delta \Delta\end{array} H Y O\right.$

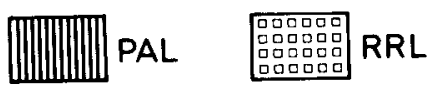

UIMGMAx

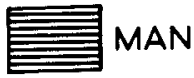

MAN

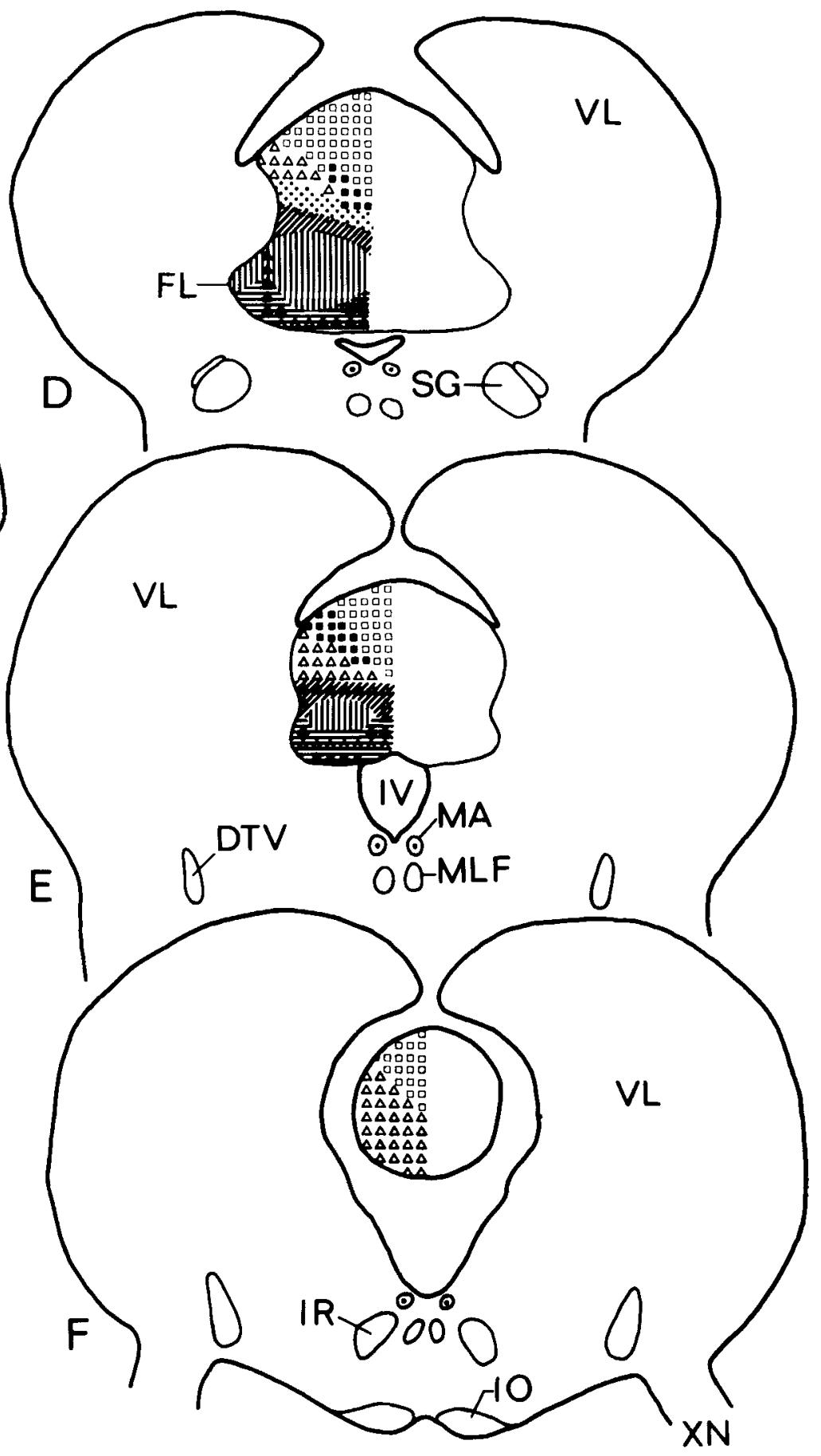



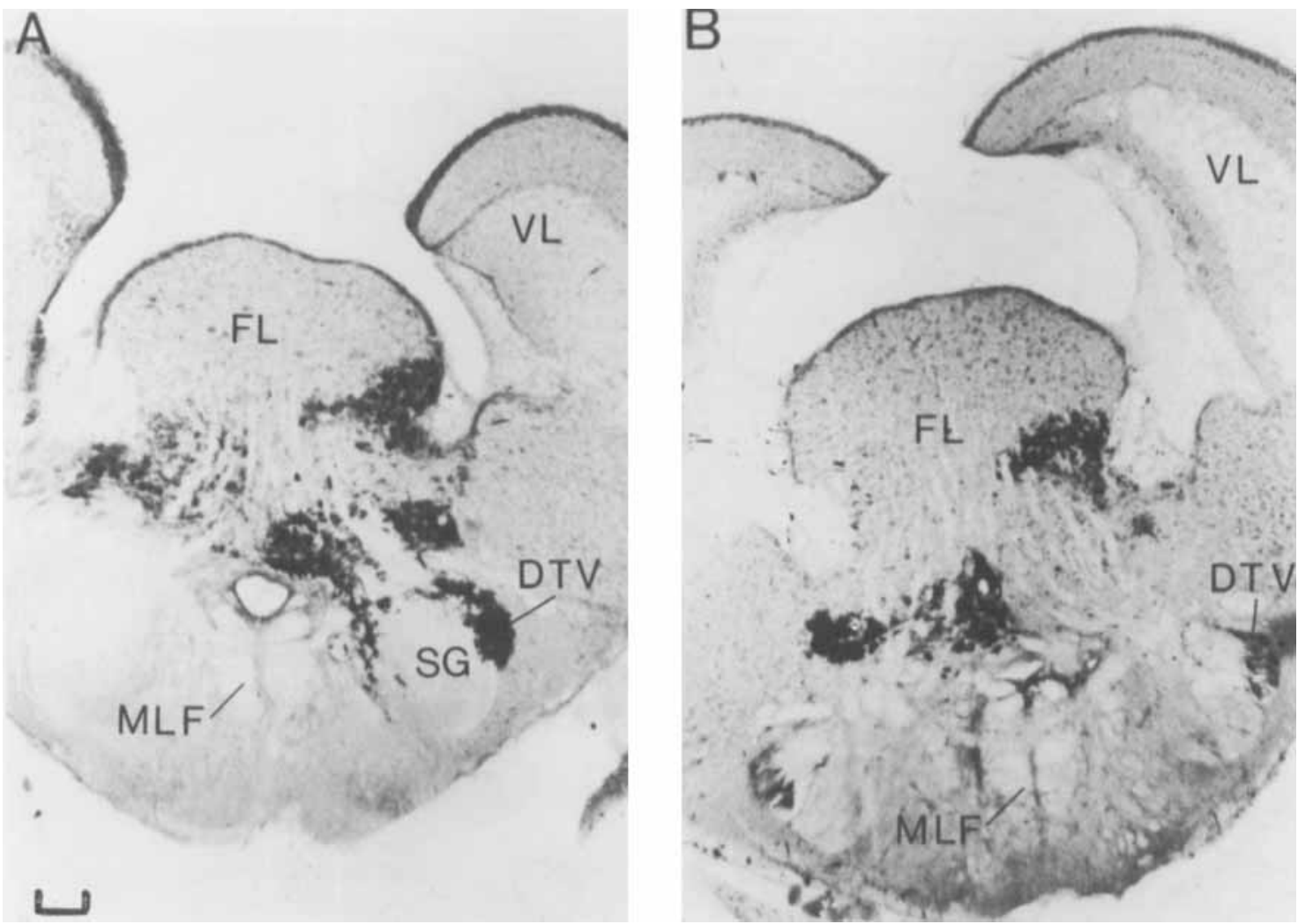

Fig. 4. Photomicrographs of transverse sections through the facial lobe (Fig. 3C,D). A: Seven days after bilateral application of HRP to the palatine ramus (left) and the infraorbital trunk (right). The figure illustrates the relations of the terminal fields of the infraorbital rami to that of the palatine ramus. B: Eight days after application of HRP to the mandibular ramus (right) and the maxillary ramus (left). Bar scale equals $0.2 \mathrm{~mm}$ for both micrographs.

the midline and project across the entire ventralmost aspect of the facial lobe (Fig. 5). In addition, at this level projections from the ventral bundles to the nucleus of descending trigeminal tract and the rostrodorsal facial motor subnucleus were observed with labeling of the entire infraorbital trunk (Fig. 5). Further, a minor projection from the descending tract of the trigeminal nerve to the ventrolateral portion of the facial lobe was observed at this level. These projections are apparent with labeling of the mandibular ramus and also appear with labeling of the maxillary ramus, although in the latter case they are extremely sparse.

The afferents of the palatine ramus enter the facial lobe through both the dorsolateral and ventral bundles of the facial sensory root and have a broad field of termination in the ipsilateral ventrointermediate portion of the lobe, located between the projection fields of the infraorbital rami (Figs. 3B-E, 4). The terminal field of the palatine ramus overlaps extensively with the projection areas of the maxillary ramus dorsally and the mandibular ramus ventrally.

Application of HRP to the hyomandibular trunk labeled fibers in the dorsolateral bundle of the facial sensory root and also lightly labeled fibers in the ventral bundles. The

Fig. 5. Photomicrograph of a transverse section through the facial lobe (Fig. 3C) 7 days after application of HRP to the infraorbital trunk. Arrows mark fibers projecting across the midline along the ventral border of the facial lobe. Arrowhead marks fibers ending among the cells of the facial motor nucleus. Asterisk marks the $\Gamma$ th ventricle. Bar scale equals $0.1 \mathrm{~mm}$.

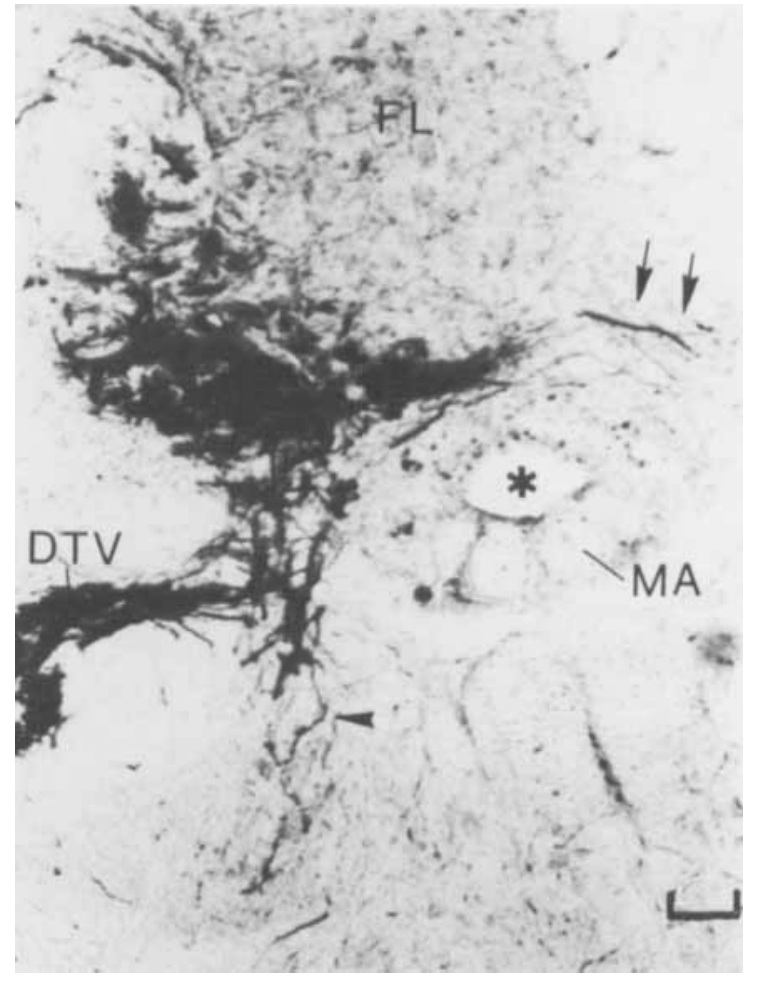



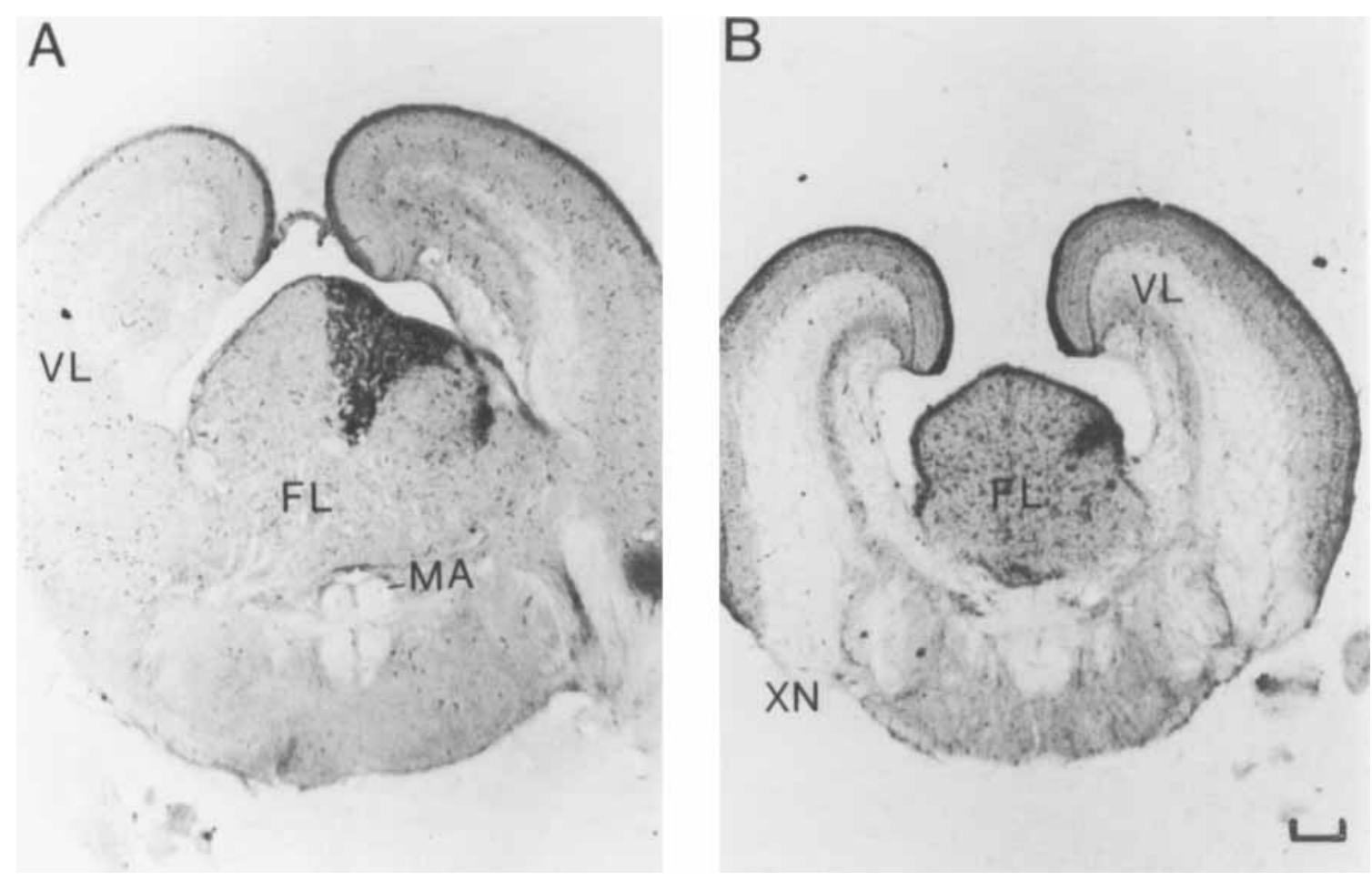

Fig. 6. Photomicrographs of transverse sections through the facial lobe (Fig. 3D,E, respectively) illustrating the terminal fields of $(\mathbf{A}:)$ the lateral recurrent ramus, 7 days after HRP application, and (B:) the pectoral recurrent ramus, 14 days after HRP application. Bar scale equals $0,2 \mathrm{~mm}$ for both micrographs.

afferent projections of the hyomandibular rami (CME, RHY, and RO, Fig. 1A) are restricted to the ipsilateral half of the facial lobe. The terminal field of this trunk begins at the level of the entrance of the rostralmost root of the vagal nerve and extends to the caudal part of the facial lobe (Fig. 3B-E). Rostrally, the terminal field of the hyomandibular rami lies dorsal to the terminal field of the superficial ramus and ventrolateral to that of the lateral recurrent ramus. Caudally, the hyomandibular terminal field lies in the ventral half of the facial lobe. There is extensive overlap with the terminal fields of the rami of the infraorbital trunk (Fig. 3D,E). Further, there is an extremely sparse projection to the nucleus of descending trigeminal tract at the same level as described for the infraorbital trunk rami.

The afferent fibers of the recurrent rami enter the facial lobe entirely from the dorsolateral bundle of the facial sensory root. The terminal projections of the recurrent rami are ipsilateral and take up the entire dorsomedial portion of the facial lobe (Figs. 3B-F, 6A,B). The terminal field of the lateral recurrent ramus extends nearly the entire length of the lobe (Fig. 3B-F). Rostrally, the terminal field of the lateral recurrent ramus shares a rostral and a ventral border with that of the superficial ophthalmic ramus. Caudally, the terminal field of the lateral recurrent ramus lies in the dorsal half of the facial lobe. The pectoral recurrent ramus has a small terminal projection field that lies along the ventrolateral edge of the terminal field of the lateral recurrent ramus. The rostralmost part of the terminal field of the pectoral recurrent ramus lies very near the center of the lobe. From this position the terminal field of this ramus extends caudolaterally between fields of the hyomandibu- lar rami and that of the lateral recurrent ramus to the dorsolateral edge of the facial lobe (Figs. 3D,E, 6B).

\section{DISCUSSION}

In addition to demonstrating the topographic organiza tion of the projections of the facial rami in the goldfish facial lobe, the present study extends in several important ways the results obtained with different techniques in the goldfish and other species of fish (Herrick, 1899, '01, 05; Luiten, '75; Morita et al., '80; Kiyohara, '85). Further, the projections of the facial sensory root in the goldfish are more extensive than what has been reported in other fishes (Finger, '76; Kiyohara et al., '85; Bartheld and Meyer, '85).

The contralateral projections observed by Luiten ('75) in the facial lobe of Cyprinis carpio following facial nerve rhizotomy were also observed in the present study. These projections are attributed to the rostral terminal fields of the facial rami which course over the dorsal head and lip regions (ROS, PAL, MAN, and MAX, Fig. 1A). Additionally, the minor projection from the descending trigeminal tract to the ventralmost aspects of the facial lobe observed by Luiten were also seen in the goldfish following labeling of the infraorbital trunk and the superficial ophthalmic ramus. The nature of this projection remains uncertain. Projections from the descending trigeminal tract to the facial lobe have also been reported in a catfish (Herrick, '06).

Projections from the facial root to the descending trigeminal tract have been reported in mammals (Rhoton, '68) and Ambystoma (Herrick, '14). Such a projection was not seen in the goldfish. However, projections to the nucleus of the 


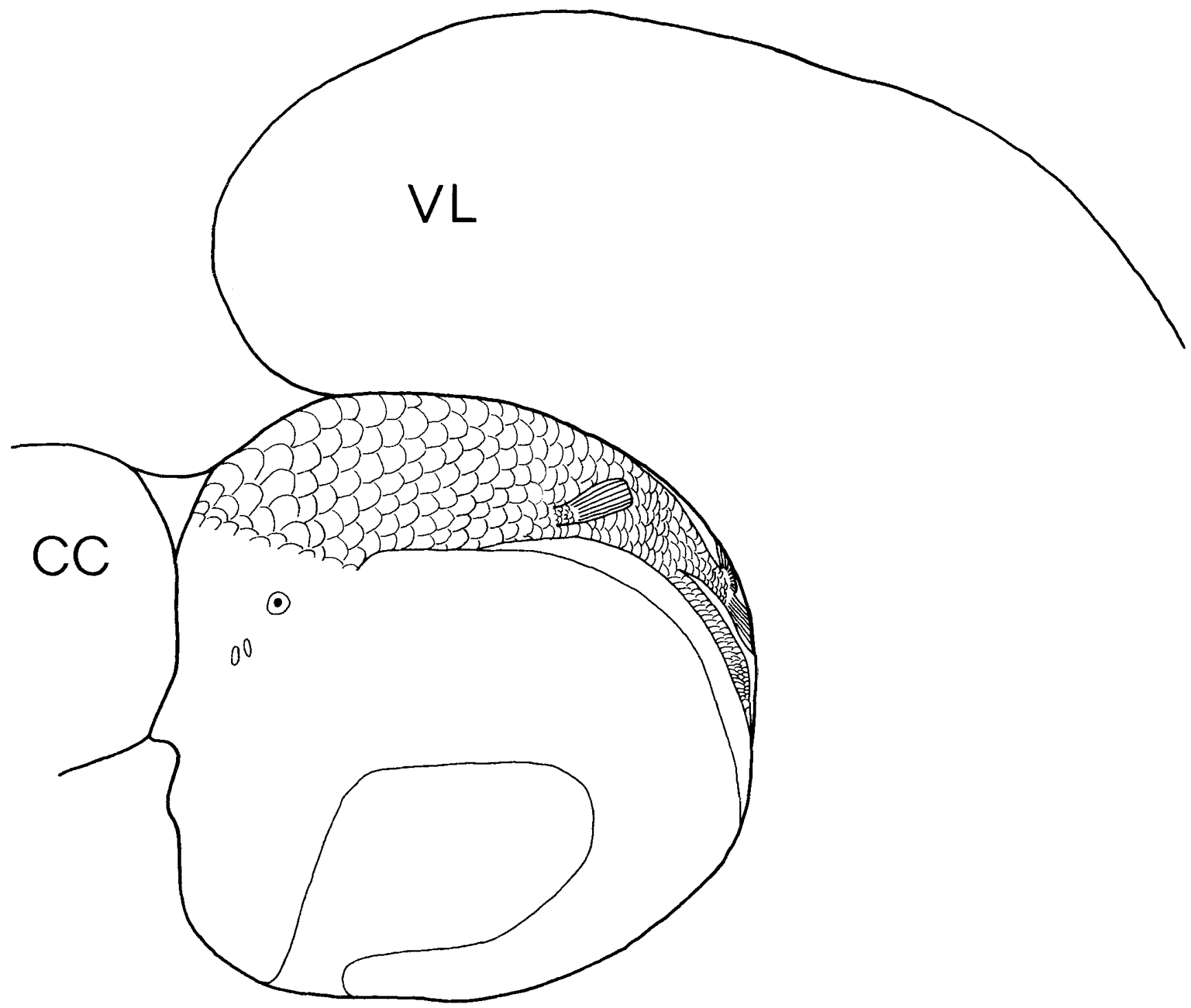

Fig. 7. Midsagittal view of the caudal hindbrain of the goldfish (Carassius auratus) with a schematic representation of the external gustatory system on the facial lobe: an pisciculus. Note the dispropor tionately large area devoted to the lips and rostral palate. Rostral is to the left.

descending trigeminal tract were observed with labeling of the infraorbital trunk, the hyomandibular trunk and the superficial ophthalmic ramus. These projections, which have not been reported previously, may represent the somatic sensory component of the facial nerve. The projections to the facial motor nucleus observed in this study may represent a monosynaptic proprioceptive circuit, which Luiten ('79) proposed based on electrophysiological recordings in the carp (C. carpio).

According to Morita et al. ('80) the sensory and recurrent roots of the facial nerve of the crucian carp (Carassius carassius) enter the brainstem separately and pursue separate courses to the facial lobe. Utilizing degeneration techniques following facial rhizotomy, Morita and co-workers found that the projections of both the sensory and recurrent roots of the facial nerve in the crucian carp were entirely ipsilateral. Further, the terminal fields of the two roots are reported to overlap entirely on the facial lobe. Based on these results, a topographic organization on the facial lobe in $C$. carassius is not distinguishable.

In contrast, the recurrent rami in the goldfish enter the brainstem along with the fibers of the other facial rami through a common root. Further, a clear topography of projections is present on the facial lobe in goldfish. The lack of a topographic organization of the projections to the facial lobe in $C$. carassius may be due to a species difference; however, this seems unlikely because of the similarity in the projection pattern reported here to that reported by Kiyohara et al. ('85) in C. carpio, a more distantly related cyprinid. It seems more probablc that the projections observed in goldfish differ from those reported in the crucian carp because of differences in techniques used.

The results of the present study, along with those of Peterson ('72), Marui ('77), and Kiyohara et al. ('85), clearly show that a topographic representation of the body axis exists on the facial lobe in cyprinids. The combined results 
of these studies suggests that the topography follows an anteroposterior orientation with the long axis of the body tilted slightly ventrally (Fig. 7). As in the catfish (Finger, '76), the lips and rostral palate take up a disproportionately large region, mapping onto nearly the entire ventral extent of the facial lobe. This disproportionately large representation of the lips and rostral palate is not surprising considering the high concentration of taste buds in these regions.

Using electrophysiological techniques to map the representation of the head region on the facial lobe of $C$. carpio, Marui (77) reported the anteroposterior body axis to be represented deep to superficial on the facial lobe. If the head and trunk axes of representation are viewed separately, it is possible to understand why Marui was led to this conclusion. The axis of the head region alone is represented dorsoventrally on the lobe, while the trunk region is represented anteroposteriorly on the lobe (Fig. 7). Thus, if one were to record from the head region alone, as Marui did, one could quite reasonably interpret a dorsoventral representation of the anteroposterior body axis.

In his electrophysiological investigation of the facial lobe in the goldfish, Peterson ('72) reported that the anteroposterior body axis is represented along the anteroposterior axis of the facial lobe. Peterson also observed only ipsilateral responses within the lobe. However, the present study demonstrates that both ipsilateral and contralateral projections exist in the goldfish facial lobe. Further, Peterson reported that there were no shifts in receptive field location with increasing depth of electrode penetration. The results presented here predict that major shifts in receptive field location should occur with increasing electrode penetration of the facial lobe. A possible explanation for these differences may be that Peterson used only tactile stimuli in his study. Physiological studies have demonstrated that the facial lobe receives both mechanoreceptive and chemoreceptive input (Marui, '77, '79). The map presented here must represent both of these types of input, while Peterson's map represents only mechanoreceptive input.

Marui and Caprio ('82) and Kiyohara et al. ('85) have argued that the somatotopy on the facial lobes in catfish is quite different from that seen in cyprinids. This may not be the case. In catfish the anteroposterior axis of the face is represented in the medial and intermediate lobules of the facial lobe in a posteroanterior axis, and the trunk is represented in the lateral lobule in an anteroposterior axis (Finger, '76; Marui and Caprio, '82). If the facial lobes of catfish were fused along the dorsal surface, so that the corresponding lobules were apposed, the medial and intermediate lobules would form the ventral portion of this lobe and the lateral lobule the dorsal portion. Thus, the trunk would be represented in the dorsal portion of the hypothetical lobe in the anteroposterior axis, and the face would be represented in the ventral portion, with minor adjustment, in the dorsoventral axis of the lobe. This would yield a condition similar to that in cyprinids.

Since little anatomical work has been done on the facial taste system of teleosts with less well developed facial lobes, comparison between the facial lobes in these teleosts and those in cyprinids and silurids is not possible. However, Bartheld and Meyer ('85) have reported that the facial fibers of the cirrus nerve in blennies (Hypsoblennius) project to a circumscribed part of the facial lobe, indicating that a facial rami somatotopy is present in fish that have relatively less well developed facial taste systems. Further anatomical work on teleosts with less well developed facial taste systems is required in order to identify the specializations that accompany the hypertrophied condition found in cyprinids and silurids.

It appears that a topographic organization of gustatory input is also present in the oropharyngeal (IXth and Xth cranial nerves) taste system. Kanwal and Caprio ('83) have provided electrophysiological evidence for a somatotopic organization of the vagal taste system in the vagal lobe of catfish. Similarly, Morita and Finger ('85) have anatomically demonstrated the presence of a viscerotopic organization of the branches of the glossopharyngeal and vagal nerves in the vagal lobe of goldfish. A gross viscerotopy has also been reported in a mormyrid fish, Gnathonemus petersii (Maler et al. '73). Additionally, a gustatory viscerotopy has been reported for mammals (Hamilton and Norgren, '84; Travers et al., '86). Thus, a topographic organization of primary gustatory input may be present in many, if not all, vertebrates.

\section{ACKNOWLEDGMENTS}

I thank Dr. Glenn Northcutt for his guidance in every aspect of the research for, and preparation of, this paper. I also thank Dr. S. Easter, Dr. B. Oakley, H. Sloan, and M.S. Northcutt for critically reading the manuscript. This research was supported in part by NIH grants NS11006 and EY02485.

\section{LITERATURE CITED}

Atema, J. (1971) Structure and functions of the sense of taste in the catfish (Ictalurus natalis). Brain Behav. Evol. 4:273-294.

Bartheld, C.S. von, and D.L. Meyer (1985) Trigeminal and facial innervation of cirri in three teleost species. Cell Tissue Res. 241:615-662.

Finger, T.E. (1976) Gustatory pathways in the bullhead catfish. I. Connections of the anterior ganglion. J. Comp. Neurol. 165:513-526.

Freihofer, W.C. (1963) Patterns of the ramus lateralis accessorius and their systematic significance in teleostean fishes. Stan. Ichthol. Bull. 8:81189.

Freihofer, W.C. (1966) The Sihler technique of staining nerves for systematic study especially of fishes. Copeia 4:470-475.

Hamilton, R.B., and R. Norgren (1984) Central projections of gustatory nerves in the rat. J. Comp. Neurol. 222:560-577.

Hanker, J.S., P.E. Yates, C.B. Metz, and A. Rustoni (1977) A new specific, sensitive and non-carcinogenic reagent for the demonstration of horseradish peroxidase. J. Histochem. 9:789-792

Herrick, C.J. (1899) The cranial and first spinal nerves of Menidia; a contribution upon the nerve components of the bony fishes. J. Comp. Neurol. $9: 153-455$

Herrick, C.J. (1901) The cranial nerves and cutaneous sense organs of the north american siluroid fishes. J. Comp. Neurol. 11:177-249.

Herrick, C.J. (1903) The organ and sense of taste in fishes. Bull. U.S. Fish Commun. 22:237-273.

Herrick, C.J. (1905) The central gustatory paths in the brains of bony fishes. J. Comp. Neurol. 15:375-456.

Herrick, C.J. (1906) On the centers for taste and touch in the medulla oblongata of fishes. J. Comp. Neurol. 16:403-439.

Herrick, C.J. (1914) The medulla oblangata of larval Amblystoma. J. Comp. Neurol. 24:343-427.

Kanwal, J.S., and J. Caprio (1983) Somatotopic mapping and characterization of visceral sensory neurons in the vagal lobe of the catfish. Soc. Neurosci. Abstr. 9:1024.

Kiyohara, S., S. Toshihiro, and S. Yamashita (1985) Periphral and central distribution of major branches of the facial taste nerve in the carp. Brain Res. 325:57-69.

Luiten, P.G.M. (1975) The central projections of the trigeminal, facial and anterior lateral line nerves in the carp (Cyprinus carpio, L.). J. Comp. Neurol. 160:399-417. 
Luiten, P.G.M. (1979) Proprioceptive reflex connections of the head musculature and the mesencephalic trigeminal nucleus in the Carp. J. Comp. Neurol. 183:903-912.

Maler, L., H.J. Karten, and M V.L. Bennett (1973) The central connections of the anterior lateral line nerve of Gnathonemus petersii. J. Comp. Neurol. 15:67-84

Marui, T. (1977) Taste responses in the facial lobe of the carp, Cyprinus carpio L. Brain Res, 130:287-298.

Marui, T. (1979) Tactile input to the facial lobe of the carp, Cyprinus carpio L. Brain Res. 177:479-488.

Marui, T., and J. Caprio (1982) Electrophysiological evidence for the topographical arrangement of taste and tactile neurons in the facial lobe of the channel catfish. Brain Res. 231:185-190.

Mesulam, M.M. (1978) Tetramethyl benzidine for horseradish peroxidase neurohistochemistry. A non-carcinogenic blue reaction-product with superior sensitivity for visualizing neural afferents and efferents. J. His tochem. Cytochem. 26:106-117.

Morita, Y., and T.E. Finger (1985) Topographic and laminar organization of the vagal gustatory system in the goldfish, Carassius auratus. J. Comp. Neurol. 238:187-201.
Morita, Y., H. Ito, and H. Masai (1980) Central gustatory paths in the crucian carp, Carassius carassius. J. Comp. Neurol, 191:119-132.

Morita, Y., T. Murakami, and H. Ito (1983) Cytoarchitecture and topographic projections of the gustatory centers in a teleost, Carassius carassius. J. Comp. Neurol. 218:378-394.

Peterson, R.H. (1972) Tactile responses of the goldfish (Carassius auratus L.) facial lobe. Copeia 3:816-819.

Puzdrowski, R.L. (1984) The primary projections of the sensory component of the facial nerve in goldfish, Carassius auratus. Soc. Neurosci. Abstr. $10: 855$.

Puzdrowski, R.L. (1985) The primary projections of the trigeminal nerve in goldfish, Carassius auratus. Soc. Neurosci. Abstr. 11:1311.

Reutter, K. (1978) Taste organ in the bullhead (Teleostei). Adv. Anat. Embryol. Cell Biol. 55:3-98.

Rhoton, A.L. (1968) Afferent connections of the facial nerve. J. Comp. Neurol. 133:89-100.

Travers, S., C. Pfaffmann, and R. Norgren (1986) Convergence of the lingual and palatal gustatory neural activity in the nucleus of the solitary tract. Brain Res. 365:305-320. 